\title{
The Complexity of Non-Monotone Markets
}

\author{
Xi Chen* \\ Columbia University \\ New York, NY \\ xichen@cs.columbia.edu
}

\author{
Dimitris Paparas ${ }^{\dagger}$ \\ Columbia University \\ New York, NY \\ paparas@cs.columbia.edu
}

\author{
Mihalis Yannakakis ${ }^{\ddagger}$ \\ Columbia University \\ New York, NY \\ mihalis@cs.columbia.edu
}

\begin{abstract}
We introduce the notion of non-monotone utilities, which covers a wide variety of utility functions in economic theory. We show that it is PPAD-hard to compute an approximate Arrow-Debreu market equilibrium in markets with linear and non-monotone utilities. Building on this result, we settle the long-standing open problem regarding the computation of an approximate Arrow-Debreu market equilibrium in markets with CES utilities, by proving that it is PPADcomplete when the Constant Elasticity of Substitution parameter, $\rho$, is any constant less than -1 .
\end{abstract}

\section{Categories and Subject Descriptors}

\section{F.2 [Analysis of algorithms and problem complexity]}

\section{Keywords}

Market equilibrium, computational complexity, CES utility, PPAD, FIXP

\section{INTRODUCTION}

General equilibrium theory $[13,20]$ is regarded by many as the crown jewel of Mathematical Economics. It studies the interactions of price, demand and supply, and is built on the demand-equal-supply principle of Walras [36]. A remarkable market model central to this field is the one of Arrow and Debreu [3], which has laid the foundation for competitive pricing mechanisms $[3,31]$.

In this model, traders exchange goods at a marketplace to maximize their utilities. ${ }^{1}$ Formally, an Arrow-Debreu market $M$ consists of a set of traders and a set of goods, denoted by $\left\{G_{1}, \ldots, G_{m}\right\}$ for some $m \geq 1$. Each trader has an initial

\footnotetext{
*Supported by NSF CCF-1149257 and a Sloan fellowship.

${ }^{\dagger}$ Supported by NSF CCF-1017955.

${ }^{\ddagger}$ Supported by NSF CCF-1017955.

${ }^{1}$ The model of Arrow and Debreu also considers firms with production. Here we focus on the setting of exchange only.
}

Permission to make digital or hard copies of all or part of this work for personal or classroom use is granted without fee provided that copies are not made or distributed for profit or commercial advantage and that copies bear this notice and the full citation on the first page. To copy otherwise, to republish, to post on servers or to redistribute to lists, requires prior specific permission and/or a fee.

STOC'13, June 1-4, 2013, Palo Alto, California, USA.

Copyright 2013 ACM 978-1-4503-2029-0/13/06 ...\$15.00. endowment $\mathbf{w} \in \mathbb{R}_{+}^{m}$, where $w_{j}$ denotes the amount of $G_{j}$ she brings to the market. Each trader also has a real-valued utility function $u$. Given a bundle $\mathbf{x} \in \mathbb{R}_{+}^{m}$ of goods, $u(\mathbf{x})$ is her utility if she obtains $\mathbf{x}$ after the exchange.

Let $\boldsymbol{\pi} \in \mathbb{R}_{+}^{m}$ denote a price vector, where $\pi_{j}$ denotes the price of $G_{j}$. Each trader first sells her initial endowment w at $\boldsymbol{\pi}$ to obtain a budget of $\mathbf{w} \cdot \boldsymbol{\pi}$. Then she spends it to buy a bundle of goods $\mathbf{x}$ from the market to maximize her utility. We say $\boldsymbol{\pi}$ is a market equilibrium of $M$, if we can assign each trader an optimal bundle, with respect to $\boldsymbol{\pi}$, such that the total demand equals the total supply and the market clears.

The celebrated theorem of Arrow and Debreu [3] asserts that under mild conditions every market has an equilibrium. Their proof, however, is based on Kakutani's fixed point theorem [25], which is non-constructive and non-algorithmic.

The problem of computing a market equilibrium was first studied in the pioneering work of Scarf [31]. During the past decade, starting with [14], the computation/approximation of market equilibria has been studied intensively, and much progress has been made. This includes efficient algorithms for various types of utility functions and market models (e.g. $[8,9,15,16,24,34,37]$ many of which are based on the convex programming approach of $[19,28])$ and complexity-theoretic results (e.g., $[4,6,10,35])$.

\section{Markets with CES Utilities}

We study the complexity of approximating market equilibria in Arrow-Debreu markets with CES (Constant Elasticity of Substitution) utilities [26].

A CES utility function takes the following form:

$$
u\left(x_{1}, \ldots, x_{m}\right)=\left(\sum_{j=1}^{m} \alpha_{j} x_{j}^{\rho}\right)^{1 / \rho}
$$

where $\alpha_{j} \geq 0$ for all $j$; and the parameter $\rho<1$ and $\rho \neq 0$.

The family of CES utilities was first introduced in [17,33]. It was used in [2] to model production functions, and predict economic growth. It has been one of the most widely used families of utility functions in economics literature [12,32], due to its versatility and flexibility in economic modeling. For example, the popular modeling language MPSGE [30] for equilibrium analysis uses CES functions (and their generalization to nested CES functions) to model consumption and production. The parameter $\rho$ of a CES utility function is related to the elasticity of substitution $\sigma$, a measure of how easy it is to substitute different goods or resources (namely, $\rho=(\sigma-1) / \sigma)$. Selecting specific values for $\rho$ between 1 and $-\infty$ yields various basic utility functions and models different points in the substitutes-complements spectrum, ranging from the perfect substitutes case when $\rho=1$, which 
corresponds to linear utilities, to the intermediate case when $\rho \rightarrow 0$, which corresponds to Cobb-Douglas utilities, and to the perfect complements case when $\rho \rightarrow-\infty$, which corresponds to Leontief utilities.

Nenakov and Primak [28] showed a convex program that characterizes the set of equilibria when $\rho=1$, i.e., all utility functions are linear. Jain [24] discovered the same convex program independently, and used the ellipsoid algorithm to give a polynomial-time exact algorithm. Indeed this convex program can also be applied to characterize the set of equilibria in CES markets, when $\rho>0$. In [7], Codenotti et al. gave a different convex formulation for the set of equilibria in CES markets with $-1 \leq \rho<0$. The range of $\rho<-1$, however, has remained an intriguing, well-known open problem. For this range, it is known that the set of market equilibria can be disconnected, and thus, one cannot hope for a direct convex formulation.

The failure of the convex-programming approach seems to suggest that the problem might be hard. Moreover, when $\rho$ $\rightarrow-\infty$, CES utilities converge to Leontief utilities for which finding an approximate market equilibrium is known to be PPAD-hard [10]. This argument, however, is less compelling because the fact that a market with CES utilities converges to a Leontief market, as $\rho \rightarrow-\infty$, does not imply that the equilibria of those CES markets converge to an equilibrium of the Leontief market at the limit, Actually, it is easy to build examples where this is not the case, and in fact, it is possible that the CES markets have equilibria that converge but the Leontief market at the limit does not even have any equilibrium or approximate equilibrium.

Moreover, with respect to the decision problem of determining whether an equilibrium exists, CES utilities do not behave like the Leontief limit but rather like the tractable utilities. Typically, the tractability of the equilibrium existence problem conforms with that of the computation problem (under standard sufficient conditions for existence). For example, both the existence problem [22] and the computation problem [24] for linear utilities can be solved in polynomial time, and the same holds for Cobb-Douglas utilities [18], whereas the existence problem is NP-hard for Leontief utilities [10] and for additively separable and piecewiselinear utilities [35] (and their computation problem is PPAD hard [4, 10,35]). However, it is known [7] that the existence problem for CES utilities is polynomial-time solvable for all (finite) values of $\rho$. This suggests that the equilibrium computation problem for CES might be also tractable.

The difficulty in resolving the complexity of CES markets with a constant parameter $\rho<-1$ is caused by the continuous nature of the problem. Most, if not all, of the problems previously shown to be PPAD-hard have a rich underlying combinatorial structure, whether it is to compute an approximate Nash equilibrium in a normal-form game [5,11] or to compute an approximate equilibrium in a market with Leontief utilities [10]; and with additively separable and concave piecewise-linear utilities $[4,35]$. In contrast, given any price vector $\boldsymbol{\pi}$, the optimal bundle $\mathbf{x}$ of a CES trader is a continuous function over $\boldsymbol{\pi}$, with an explicit algebraic form (see (2) in Section 2). The problem of finding a market equilibrium then boils down to solving a system of polynomial equations over variables $\boldsymbol{\pi}$, and it is not clear how to extract from it a useful combinatorial structure.

Our first result resolves the complexity of approximating equilibria in CES markets for all values of $\rho<-1$ :
TheOREM 1. For any fixed rational number $\rho<-1$, the problem of finding an approximate market equilibrium in a CES market of parameter $\rho$ is PPAD-complete.

It is worth mentioning here that the notion of approximate market equilibria used in Theorem 1 is one-sided, i.e., $\boldsymbol{\pi}$ is an $\epsilon$-approximate market equilibrium if the excess demand of each good is bounded from above by $\epsilon$-fraction of the total supply. While the two-sided notion is more commonly used in the literature (which we refer to as $\epsilon$-tight approximate market equilibria), i.e., the absolute value of excess demand is bounded, we present an unexpected CES market example with $\rho<0$ in Section 2.2 and show that any of its $1 / 2$-tight approximate equilibria requires exponentially many bits to describe. By contrast, we show that for the one-sided notion there is always an $\epsilon$-approximate equilibrium with a polynomial number of bits, and, furthermore, its computation is in PPAD. We show also that the problem of computing an actual equilibrium (to any desired precision) is in FIXP.

\section{Non-Monotone Families of Utilities}

The resolution of the complexity of CES markets with parameter $\rho<-1$ inspired us to ask the following question:

$$
\begin{gathered}
\text { Can we prove a complexity dichotomy } \\
\text { for any given family of utility functions? }
\end{gathered}
$$

Formulating it more precisely, we use $\mathcal{U}$ to denote a generic family of functions that satisfy certain mild conditions (e.g., they should be continuous and quasi-concave).

The question now becomes the following: Does there exist a mathematically well-defined property on families of functions such that: For any $\mathcal{U}$ satisfying this property, the equilibrium problem it defines is in polynomial time; For any $\mathcal{U}$ that violates this property, the problem is hard.

For the algorithmic part of this question, a property that has played a critical role in the approximation of market equilibria is WGS (Weak Gross Substitutability). Here a family $\mathcal{U}$ of utilities satisfies WGS if, for any market consisting of traders with utilities from $\mathcal{U}$, increasing the prices of some goods while keeping all other prices fixed cannot cause a decrease in the demand of any good with fixed price. WGS implies that the set of equilibrium prices is convex, the classical tatonnement process always converges to an equilibrium [1] and a discrete version of tatonnement [8] converges in polynomial time to an approximate equilibrium. Another general property that also implies convexity of the set of equilibria is WARP (Weak Axiom of Revealed Preference, see [26] for definition and background).

While several families of utilities satisfy WGS or WARP, they do not cover all efficiently solvable market problems, e.g., the family of CES utilities with parameter $\rho:-1 \leq$ $\rho<0$ does not satisfy WGS or WARP, but has a convex formulation after a change of variables [7].

For the hardness part of this question, our knowledge is much more limited. Only for a few specific and isolated families of utilities mentioned earlier, is the problem of finding an approximate equilibrium shown to be hard. And the reduction techniques developed in these proofs are all different, each fine tuned for the utilities being considered.

Our second contribution is a PPAD-hardness result that is widely applicable to any generic family of utility functions as long as it satisfies the following condition: 
DEFINITION 1.1. (INFORMAL). $\mathcal{U}$ is a non-monotone family of utilities if there exists a market $M$ with utilities from $\mathcal{U}$, a price vector $\boldsymbol{\pi}$, and a good $G$ of $M$, such that: 1) the excess demand of good $G$ at $\boldsymbol{\pi}$ is nonnegative, and 2) raising the price of $G$ at $\boldsymbol{\pi}$, while keeping all other prices the same, (strictly) increases the demand of $G$.

Examples of non-monotone families include CES with $\rho<$ -1 , Leontief, and separable, piecewise linear functions (see Section 2.3). Our second result shows that non-monotonicity implies the following hardness result:

TheOREM 2. (INFORMAL). If $\mathcal{U}$ is non-monotone, then the following problem is PPAD-hard: Given a market in which the utility of each trader is either linear or from $\mathcal{U}$, find an approximate equilibrium.

We remark that there is clearly a gap between WGS and non-monotonicity. It remains an open problem as whether we can further reduce the gap, and whether we can remove the use of linear functions in Theorem 2.

The rest of the paper is organized as follows. In Section 2, we give basic definitions and state formally our main results. Sections 3 and 4 give the PPAD-hardness proofs (Theorems 2 and 1 ). Sections 5 shows that the problem of computing an equilibrium for CES markets is in FIXP and Section 6 shows that computing an approximate equilibrium is in PPAD.

\section{PRELIMINARIES AND MAIN RESULTS}

Notation. $\mathbb{R}_{+}$is the set of nonnegative real numbers; $\|\cdot\|$ denotes the $L_{\infty}$ norm. Given a vector $\mathbf{y} \in \mathbb{R}^{m}$ and $c>0$, we use $B(\mathbf{y}, c)$ to denote the set of $\mathbf{x}$ with $\|\mathbf{x}-\mathbf{y}\| \leq c$.

\subsection{Markets and Market Equilibria}

An Arrow-Debreu market $M$ consists of a set of traders, $\left\{T_{1}, \ldots, T_{n}\right\}$ and a set of goods, $\left\{G_{1}, \ldots, G_{m}\right\}$. Each trader $T_{i}$ has an initial endowment $\mathbf{w}_{i} \in \mathbb{R}_{+}^{m}$, where $w_{i, j}$ denotes the amount of good $G_{j}$ she initially owns, and a utility function $u_{i}: \mathbf{R}_{+}^{m} \rightarrow \mathbf{R}_{+}$, where $u_{i}\left(x_{i, 1}, \ldots, x_{i, m}\right)$ represents the utility she derives if the amount of $G_{j}$ she obtains by the end is $x_{i, j}$ for each $j \in[m]$.

Let $\boldsymbol{\pi}=\left(\pi_{1}, \ldots, \pi_{m}\right) \neq \mathbf{0}$ be a nonnegative price vector, where $\pi_{j} \geq 0$ denotes the price per unit of $G_{j}$. Each trader $T_{i}$ sells her initial endowment $\mathbf{w}_{i}$ at prices $\boldsymbol{\pi}$ and obtains a budget of $\mathbf{w}_{i} \cdot \boldsymbol{\pi}=\sum_{j \in[m]} w_{i, j} \cdot \pi_{j}$. She then spends the budget to buy a bundle of goods $\mathbf{x}_{i} \in \mathbb{R}_{+}^{m}$ from the market to maximize her utility.

Let $\mathrm{opt}_{i}(\boldsymbol{\pi})$ denote the set of optimal bundles (demands) of $T_{i}$ with respect to $\boldsymbol{\pi}$, i.e., the set of vectors $\mathbf{x} \in \mathbb{R}_{+}^{m}$ that maximize $u_{i}(\mathbf{x})$ subject to $\mathbf{x} \cdot \boldsymbol{\pi} \leq \mathbf{w}_{i} \cdot \boldsymbol{\pi}$. The (aggregate) excess demand $Z(\boldsymbol{\pi})$ consists of all the vectors $\mathbf{z}$ of the form $\mathbf{z}=\mathbf{x}_{1}+\cdots+\mathbf{x}_{m}-\left(\mathbf{w}_{1}+\cdots+\mathbf{w}_{m}\right)$, where $\mathbf{x}_{i} \in \operatorname{opt}_{i}(\boldsymbol{\pi})$ for each $i \in[n]$. For each $G_{j}$, we use $Z_{j}(\boldsymbol{\pi})$ to denote the projection of $Z(\boldsymbol{\pi})$ on the $j$ th coordinate. In general, $Z(\boldsymbol{\pi})$ is a set and $Z$ is a correspondence. Also note that given a $\mathbf{z} \in Z(\boldsymbol{\pi})$, when $z_{j}>0$ the traders demand more of $G_{j}$ than the total available supply; when $z_{j}<0$ they demand less.

We say $\boldsymbol{\pi}$ is a market equilibrium of $M$ if we can assign each trader $T_{i}$ an optimal bundle $\mathbf{x}_{i}$ with respect to $\boldsymbol{\pi}$, such that the total demand equals the total supply and the market clears, i.e. $\boldsymbol{\pi}$ is a market equilibrium iff $\mathbf{0} \in Z(\boldsymbol{\pi})$. Since $\operatorname{opt}_{i}(\boldsymbol{\pi})$ is invariant under scaling of $\boldsymbol{\pi}$ (by a positive factor), the set of market equilibria is closed under scaling.
Let $W_{i}=\sum_{i \in[n]} w_{i, j}$ denote the total supply of $G_{j}$. Now we define two versions of approximate market equilibria:

Definition 1. We call $\boldsymbol{\pi}$ an $\epsilon$-approximate market equilibrium of $M$, for some $\epsilon>0$, if there exists a vector $\mathbf{z} \in Z(\boldsymbol{\pi})$ such that $z_{j} \leq \epsilon W_{j}$ for all $j \in[m]$.

We call $\boldsymbol{\pi}$ an $\epsilon$-tight approximate market equilibrium if there exists $\mathbf{z} \in Z(\boldsymbol{\pi})$ such that $\left|z_{j}\right| \leq \epsilon W_{j}$ for all $j$.

Both notions of approximate equilibria have been used in the literature. While the two-sided notion is more commonly used, we present a CES market with $\rho<0$ in Section 2.2, of which every $1 / 2$-tight approximate equilibrium requires an exponential number of bits to describe.

In general, a market equilibrium may not exist. The pioneering theorem of Arrow and Debreu [3] asserts that under certain mild conditions a market always has an equilibrium. Here we use the weaker sufficient condition of Maxfield [27]. We say a utility function $u: \mathbb{R}_{+}^{m} \rightarrow \mathbb{R}_{+}$is non-satiated with respect to the $k$ th good, if for any $\mathbf{x} \in \mathbb{R}_{+}^{m}$, there exists a $\mathbf{y} \in \mathbb{R}_{+}^{m}$ such that $u(\mathbf{y})>u(\mathbf{x})$ and $y_{j}=x_{j}$ for all $j \neq k$. We say $u$ is locally non-satiated if for any vector $\mathbf{x} \in \mathbb{R}_{+}^{m}$ and any $\epsilon>0$, there exists a $\mathbf{y} \in B(\mathbf{x}, \epsilon)$ such that $u(\mathbf{y})>u(\mathbf{x})$. If the utility of a trader is locally non-satiated then her optimal bundle must exhaust her budget. Hence, if every trader in $M$ has a locally non-satiated utility, then Walras' law holds: $\mathbf{z} \cdot \boldsymbol{\pi}=0$ for all $\mathbf{z} \in Z(\boldsymbol{\pi})$.

From a market $M$, we can define a directed graph, called the economy graph of $M$, which has a vertex for each good and has an edge from $G_{i}$ to $G_{j}$ if there is a trader $T_{k}$ such that $w_{k, i}>0$ and $u_{k}$ is non-satiated with respect to $G_{j}$. We call $M$ strongly connected if its economy graph is. Maxfield [27] showed that if $M$ is strongly connected and all utilities are continuous, quasi-concave, and locally non-satiated, then $M$ always has an equilibrium. (Maxfield used a graph with the traders as vertices but the two graphs are related.)

\subsection{CES Utility Functions}

We call $u$ a CES function with parameter $\rho<1, \rho \neq 0$, if it is of the form given in (1). Let $T$ denote a trader with a CES utility $u$, initial endowment $\mathbf{w}$, and let $S=\{j \in[m]$ : $\left.\alpha_{j}>0\right\}$ be the goods in which she is interested. If $\boldsymbol{\pi}>\mathbf{0}$ is a positive price vector, then using the KKT conditions one can show that $T$ has a unique optimal bundle $\mathbf{x}$ where

$$
x_{j}=\left(\frac{\alpha_{j}}{\pi_{j}}\right)^{1 /(1-\rho)} \times \frac{\mathbf{w} \cdot \boldsymbol{\pi}}{\sum_{k \in S} \alpha_{k}^{1 /(1-\rho)} \cdot \pi_{k}^{-\rho /(1-\rho)}}
$$

for each $j \in S$. When a CES market $M$ is strongly connected, then $\pi_{j}$ must be positive, for all $j \in[m]$, in any (exact or approximate) market equilibrium of $M$.

The problem of whether there exists an equilibrium in a CES market can be solved in polynomial time: a simple necessary and sufficient condition for the existence of an equilibrium was shown in [7] based on the decomposition of the economy graph into strongly connected components. They furthermore showed that the computation of an equilibrium for the whole market (if the condition is satisfied) reduces to the computation of equilibria for the submarkets induced by the strongly connected components. Thus, we will focus on strongly connected markets.

As a CES market may not have a rational equilibrium in general, even when $\rho$ and all the coefficients are rational, we 
study the approximation of market equilibria. To this end, we define the following three problems:

1. CES: The input of the problem is a pair $(k, M)$, where $k$ is a positive integer encoded in unary ( $k$ represents the desired number of bits of precision), and $M$ is a strongly connected market in which all utilities are CES, with the parameter $\rho_{i}<1$ of each trader $T_{i}$ being rational and given in unary (because $\rho$ appears in the exponent in the utility and demand functions). Both the endowments $w_{i, j}$ and coefficients $\alpha_{i, j}$ are rational and encoded in binary. The goal is to find a price vector $\pi$ that is within $1 / 2^{k}$ of some equilibrium in every coordinate, i.e., such that there exists an (exact) equilibrium $\boldsymbol{\pi}^{*}$ of $M$ with $\left\|\boldsymbol{\pi}-\boldsymbol{\pi}^{*}\right\|_{\infty} \leq 1 / 2^{k}$.

2. CES-APPROX: The input is the same as CES. The goal is to find an $\epsilon$-approximate market equilibrium, $\epsilon=1 / 2^{k}$.

3. For each (fixed) rational number $\rho<-1$, we also define the following problem $\rho$-CES-APPROX: The input is the same as CES, except that the utilities of all the traders have the same fixed parameter $\rho$, which is considered as a constant, not part of the input. The goal is to find an $\epsilon$-approximate market equilibrium of $M$, where $\epsilon=1 / k$.

Next we present the following example to justify the use of $\epsilon$-approximate market equilibria, instead of the two-sided $\epsilon$-tight approximate market equilibria:

EXAMPLE 2.1. Fix $a \rho<0$ and let $r=|\rho|>0$. Let $M$ denote the following CES market with parameter $\rho$. Here $M$ has $n$ goods $G_{1}, \ldots, G_{n}$ and $n$ traders $T_{1}, \ldots, T_{n}$. Each $T_{i}, i \in[n]$, has $2^{i(n+1)}$ units of good $G_{i}$ at the beginning. Each $T_{i}, i \in[n-1]$, is equally interested in $G_{1}$ and $G_{i+1}$ so in particular, $T_{1}$ is interested in only $G_{1}$ and $G_{2} . T_{n}$ is only interested in $G_{1}$. The economy graph of $M$ is clearly strongly connected. We prove in the full version that any (1/2)-tight approximate equilibrium $\boldsymbol{\pi}$ must have

$$
\max _{j} \pi_{j} / \min _{j} \pi_{j} \geq 2^{n(1+r)^{n-2}}
$$

We state our main results. First in Section 5 and Section 6, we show the membership of CES in FIXP [21] and the membership of CES-APPROX in PPAD [29], respectively:

\section{THEOREM 3. CES is in FIXP.}

Theorem 4. CES-APPRox is in PPAD.

We prove in Section 4 that CES markets are PPAD-hard to solve when $\rho<-1$. Combined with Theorem 4 , we have:

THEOREM 5. For any rational number $\rho<-1$, the problem $\rho$-CES-APPROX is PPAD-complete.

The hard instances we construct in the proof of Theorem 5 are in fact very restricted in the sense that each trader is interested in either one or two goods and applies one of the following utility functions: $u(x)=x, u\left(x_{1}, x_{2}\right)=\left(x_{1}^{\rho}+\right.$ $\left.x_{2}^{\rho}\right)^{1 / \rho}$, or $u\left(x_{1}, x_{2}\right)=\left(\alpha \cdot x_{1}^{\rho}+x_{2}^{\rho}\right)^{1 / \rho}$, where $\alpha$ is a positive rational constant that depends on $\rho$ only.

\subsection{Non-Monotone Markets and Utilities}

We use $\mathcal{U}$ to denote a generic family of continuous, quasiconcave and locally non-satiated functions, e.g., linear functions, piecewise-linear functions (see Example 2.4), CES functions for a specific parameter of $\rho$, e.g. $\rho=-3$, or even the finite set of three functions given above. Ideas behind the proof of Theorem 5 allow us to prove a PPAD-hardness result for approximating equilibria of markets in which the utility of each trader is either linear or from $\mathcal{U}$, when $\mathcal{U}$ is "non-monotone". We first formally set up the problem.

First, we assume that $\mathcal{U}$ is countable and each function $g \in \mathcal{U}$ corresponds to a unique binary string, so that a trader can specify a function $g \in \mathcal{U}$ using a binary string. In a market with $m$ goods, we say a trader "applies" a function $g \in \mathcal{U}$ if her utility function $u$ is of the form $u\left(x_{1}, \ldots, x_{m}\right)=$ $g\left(x_{\ell_{1}} / b_{1}, \ldots, x_{\ell_{k}} / b_{k}\right)$ where $g \in \mathcal{U}$ has $k \leq m$ variables; $\ell_{1}, \ldots, \ell_{k} \in[m]$ are distinct indices; and $b_{1}, \ldots, b_{k}$ are positive rational numbers (representing a setting of units for the goods by scaling). In this way, each trader can be described by a finite binary string. Second, we assume that there exists a univariate function $g^{*} \in \mathcal{U}$ that is strictly monotone (i.e., can have single-minded traders that are interested in only one good). We use $\mathcal{M}_{\mathcal{U}}$ to denote the set of all markets in which every trader has a rational initial endowment and applies a utility function from $\mathcal{U}$. We also use $\mathcal{M}_{\mathcal{U}}^{*}$ to denote the set of markets in which every trader has a rational initial endowment and applies either a utility function from $\mathcal{U}$ or a linear utility with rational coefficients.

We define non-monotone markets and families of utilities:

Definition 2. Let $M$ be a market with $k \geq 2$ goods. We say $M$ is non-monotone at a price vector $\boldsymbol{\pi}>\mathbf{0}$ if for some $c>0$ and some good in $M$, say $G_{1}$, the excess demand $Z_{1}\left(y_{1}, \ldots, y_{k}\right)$ of $G_{1}$ is a continuous function (instead of a correspondence) over $\mathbf{y} \in B(\boldsymbol{\pi}, c)$, with $Z_{1}(\boldsymbol{\pi}) \geq 0$; the partial derivative of $Z_{1}$ with respect to $y_{1}$ exists in $B(\boldsymbol{\pi}, c)$, is continuous over $B(\boldsymbol{\pi}, c)$, and is (strictly) positive at $\boldsymbol{\pi}$.

We call $M$ a non-monotone market if there exists such a price vector $\boldsymbol{\pi}>\mathbf{0}$. We call $\mathcal{U}$ a non-monotone family of utilities if there exists a non-monotone market in $\mathcal{M U}_{\mathcal{U}}$.

By definition, $M$ being non-monotone at $\boldsymbol{\pi}$ means that raising the price of $G_{1}$ while keeping the prices of all other goods the same would actually increase the total demand of $G_{1}$. It is easy to see, using the continuity of $Z_{1}$ and its partial derivative $\partial Z_{1} / \partial y_{1}$ in $B(\boldsymbol{\pi}, c)$, that we can require, without loss of generality, the price vector $\pi$ to be rational in Definition 2. However, $Z_{1}(\boldsymbol{\pi})$ and $\left(\partial Z_{1} / \partial y_{1}\right)(\boldsymbol{\pi})$ do not have to be rational. Also, $M$ is not necessarily strongly connected. In our hardness proofs we will use the market $M$ and price $\boldsymbol{\pi}$ of the definition as a black-box gadget. Although the size of the gadget market can be treated as a constant (it only depends on the family $\mathcal{U}$ ), we will need to compute approximately $Z_{1}(\boldsymbol{\pi})$ to desired precision. We say a real number $\beta$ is moderately computable if there is an algorithm that, given $\gamma>0$, outputs a $\gamma$-rational approximation $\beta^{\prime}$ of $\beta:\left|\beta^{\prime}-\beta\right| \leq \gamma$, in time polynomial in $1 / \gamma$.

Now we can state our PPAD-hardness result for a nonmonotone family $\mathcal{U}$ of functions. We use $\mathcal{U}$-market to denote the following problem: The input is a pair $(k, M)$, where $k$ is a positive integer in unary and $M$ is a strongly connected market from $\mathcal{M}_{\mathcal{U}}^{*}$ encoded in binary. The goal is to output an $\epsilon$-approximate equilibrium of $M$ with $\epsilon=1 / k$.

THEOREM 6. Let $\mathcal{U}$ be a non-monotone family of utility functions. If there exists a market $M \in \mathcal{M U}_{\mathcal{U}}$ such that $M$ is non-monotone at a rational price vector $\boldsymbol{\pi}>\mathbf{0}$ and the excess demand $Z_{1}(\boldsymbol{\pi})$ of $G_{1}$ at $\boldsymbol{\pi}$ is moderately computable, then the problem $\mathcal{U}$-market is PPAD-hard. 
Next, we give three examples of non-monotone markets.

EXAmple 2.2. (CES Utilities With $\rho<-1$ ) Consider the following market $M$ with two goods $G_{1}$ and $G_{2}$, and two traders $T_{1}$ and $T_{2} . T_{1}$ has one unit of $G_{1}, T_{2}$ has one unit of $G_{2}$, and the utilities are $u_{1}\left(x_{1}, x_{2}\right)=\left(\alpha x_{1}^{\rho}+x_{2}^{\rho}\right)^{1 / \rho}$ and $u_{2}\left(x_{1}, x_{2}\right)=\left(x_{1}^{\rho}+\alpha x_{2}^{\rho}\right)^{1 / \rho}$, respectively.

When $\rho<-1$ and $\alpha$ is large enough, [23] proves that $M$ has $(1,1)$ as an equilibrium and is non-monotone at $(1,1)$. This implies that $M$ has multiple isolated equilibria, and the set of equilibria of a CES market with $\rho<-1$ is not convex (not even connected) in general.

Example 2.3. (LeOntief Utilities) We say $u$ is a Leontief utility function if $u\left(x_{1}, \ldots, x_{k}\right)=\min _{j \in S}\left\{x_{j} / a_{j}\right\}$, where $a_{j}>0$ for all $j \in S \subseteq[k]$. Let $M$ be the Leontief market consisting of the following two traders $T_{1}$ and $T_{2}$. Here $T_{1}$ has one unit of $G_{1}$ and $T_{2}$ has one unit of $G_{2}$. The utility function of $T_{1}$ is $u_{1}\left(x_{1}, x_{2}\right)=\min \left\{x_{1} / 2, x_{2}\right\}$; the utility function of $T_{2}$ is $u_{2}\left(x_{1}, x_{2}\right)=\min \left\{x_{1}, x_{2} / 2\right\}$. Then $M$ is non-monotone at $(1,1)$.

Example 2.4. (Separable, Piecewise-Linear Utilities). A utility function is additively separable and piecewise linear if $u\left(x_{1}, \ldots, x_{k}\right)=f_{1}\left(x_{1}\right)+\cdots+f_{k}\left(x_{k}\right)$, where $f_{1}, \ldots, f_{k}$ are piecewise-linear functions. Consider the following market $M$ with two goods $G_{1}, G_{2}$, and two traders $T_{1}, T_{2}$. $T_{1}$ has one unit of $G_{1}$, and $T_{2}$ has one unit of $G_{2}$. Their utility functions are $u_{1}\left(x_{1}, x_{2}\right)=x_{1}+f\left(x_{2}\right)$ and $u_{2}\left(x_{1}, x_{2}\right)=f\left(x_{1}\right)+x_{2}$, with $f(x)=2 x$ if $x \leq 1 / 3$; and $f(x)=2 / 3$ if $x>1 / 3$. It can be shown that $M$ has $(1,1)$ as an equilibrium and is non-monotone at $(1,1)$.

Note that in general, the excess demand of a market with such utilities is a correspondence instead of a map, and partial derivatives may not always exist. But in our definition of non-monotone markets, we only need those properties in a local neighborhood of $\boldsymbol{\pi}$, like $(1,1)$ here.

Since linear functions are special cases of additively separable, piecewise-linear functions, we obtain a corollary from Theorem 6 and Example 2.4: finding an approximate equilibrium in a market with additively separable, concave, and piecewise-linear utilities is PPAD-hard. This is the main result of [4]. Combining it with the PPAD membership of [35]:

COROLlary 2.1. The problem of finding an approximate equilibrium in a market with additively separable, concave, and piecewise-linear utilities is PPAD-complete, even when each univariate function is either linear or of the form of $f$ in Example 2.4, i.e., linear function with a threshold.

\subsection{Polymatrix Games and Nash Equilibria}

To prove Theorem 5 and 6 , we give polynomial-time reductions from the problem of finding an approximate Nash equilibrium in a polymatrix game, with two pure strategies for each player. Such a game with $n$ players can be described by a $2 n \times 2 n$ rational matrix $\mathbf{P}$, with all entries between 0 and 1. An $\epsilon$-well-supported Nash equilibrium is then a vector $\mathbf{x} \in \mathbb{R}_{+}^{2 n}$ such that $x_{2 i-1}+x_{2 i}=1$ and

$$
\begin{aligned}
& \mathbf{x}^{T} \cdot \mathbf{P}_{2 i-1}>\mathbf{x}^{T} \cdot \mathbf{P}_{2 i}+\epsilon \Rightarrow x_{2 i}=0 \\
& \mathbf{x}^{T} \cdot \mathbf{P}_{2 i}>\mathbf{x}^{T} \cdot \mathbf{P}_{2 i-1}+\epsilon \Rightarrow x_{2 i-1}=0
\end{aligned}
$$

for all $i \in[n]$, where we use $\mathbf{P}_{2 i-1}$ and $\mathbf{P}_{2 i}$ above to denote the $(2 i-1)$ th and $(2 i)$ th column vectors of $\mathbf{P}$, respectively.
We let POLYMATRIX denote the following problem: Given a polymatrix game $\mathbf{P}$, find an $\epsilon$-well-supported Nash equilibrium with $\epsilon=1 / n$. It was shown in [11] that computing an exact Nash equilibrium of a polymatrix game with two strategies for each player is PPAD-complete. We show in the full paper that POLYMATRIX is also PPAD-hard. The proof follows closely techniques developed in $[5,11]$.

TheOrem 7. POlymatrix is PPAD-complete.

\section{PPAD-HARDNESS FOR NON-MONOTONE UTILITIES}

We start with a brief sketch of our construction. Given a $2 n \times 2 n$ polymatrix game $\mathbf{P}$, we build a market $M_{\mathbf{P}} \in \mathcal{M}_{\mathcal{U}}^{*}$, and prove that, given any $\epsilon$-approximate equilibrium of $M_{\mathbf{P}}$ for some polynomially small $\epsilon$, we can recover a $(1 / n)$-wellsupported Nash equilibrium $\mathbf{x}$ of $\mathbf{P}$ in polynomial time.

A building block of $M_{\mathbf{P}}$ is the linear price-regulating markets $[4,35]$. Such a market consists of two traders $\left\{T_{1}, T_{2}\right\}$ and two goods $\left\{G_{1}, G_{2}\right\}$. Each $T_{i}$ owns $\tau>0$ units of $G_{i}$, $i \in\{1,2\}$. The utility of $T_{1}$ is $(1+\alpha) x_{1}+(1-\alpha) x_{2}$ and the utility of $T_{2}$ is $(1-\alpha) x_{1}+(1+\alpha) x_{2}$, for some $\alpha \in(0,1)$.

Let $\pi_{i}$ denote the price of $G_{i}$. Then we have the following useful property: Even if we add more traders to the market, as long as their total endowment of $G_{1}$ and $G_{2}$ is negligible compared to $\tau$, the ratio $\pi_{1} / \pi_{2}$ must be between $(1-\alpha) /(1+$ $\alpha)$ and $(1+\alpha) /(1-\alpha)$ at an approximate equilibrium.

Our construction then starts with the following blueprint of encoding $2 n$ variables $\mathbf{x}$ and the $2 n$ linear forms $\mathbf{x}^{T} \cdot \mathbf{P}_{j}$, $j \in[2 n]$, in $M_{\mathbf{P}}$. Let $G_{1}, \ldots, G_{2 n}$ and $H_{1}, \ldots, H_{2 n}$ denote $4 n$ goods. Let $\tau=n^{2}$. Let $\alpha$ and $\beta$ be two polynomially small parameters with $\alpha \ll \beta$. For each $i \in[n]$, we first create a price-regulating market over $G_{2 i-1}$ and $G_{2 i}$ with parameters $\tau$ and $\alpha$, and a price-regulating market over $H_{2 i-1}$ and $H_{2 i}$, with $\tau$ and $\beta$. For each $i, j \in[2 n]$, we add a trader $T_{i, j}$ who owns $P_{i, j}$ units of $H_{i}$ and is only interested in $G_{j}$.

At this moment, the property of price-regulating markets implies that at any approximate equilibrium $\boldsymbol{\pi}$, the ratio of $\pi\left(H_{2 i-1}\right)$ and $\pi\left(H_{2 i}\right)$ is in $[(1-\beta) /(1+\beta),(1+\beta) /(1-\beta)]$; the ratio of $\pi\left(G_{2 i-1}\right)$ and $\pi\left(G_{2 i}\right)$ is in $[(1-\alpha) /(1+\alpha),(1+$ $\alpha) /(1-\alpha)$ ], where we use $\pi(G)$ to denote the price of a good $G$ in $\boldsymbol{\pi}$. Here is some wishful thinking: If for every $i \in[n]$, we have $\pi\left(H_{2 i-1}\right)+\pi\left(H_{2 i}\right)=\pi\left(G_{2 i-1}\right)+\pi\left(G_{2 i}\right)=2$, then a vector $\mathbf{x}$ can be extracted from $\boldsymbol{\pi}$ as follows:

$$
x_{i}=\frac{\pi\left(H_{i}\right)-(1-\beta)}{2 \beta}, \text { for each } i \in[2 n] .
$$

Clearly $\mathbf{x}$ is nonnegative, and satisfies $x_{2 i-1}+x_{2 i}=1$ for all $i$. The $2 n$ linear forms $\mathbf{x}^{T} \cdot \mathbf{P}_{j}$ we are interested in now appear in $M_{\mathbf{P}}$ as follows: The total money that traders $T_{i, j}$, $i \in[2 n]$, spend on good $G_{j}$ is

$$
\sum_{i \in[2 n]} P_{i, j}\left(2 \beta x_{i}+(1-\beta)\right)=2 \beta \cdot \mathbf{x}^{T} \cdot \mathbf{P}_{j}+(1-\beta) \sum_{i \in[2 n]} P_{i, j}
$$

With some wishful thinking, assume all the $\mathbf{P}_{j}$ 's sum to the same value. As $\beta \gg \alpha, \mathbf{x}^{T} \cdot \mathbf{P}_{2 j-1}>\mathbf{x}^{T} \cdot \mathbf{P}_{2 j}+1 / n$ would imply that the total demand for $G_{2 j-1}$ from traders $T_{i, 2 j-1}$, $i \in[2 n]$, must be strictly larger than that of $G_{2 j}$ from $T_{i, 2 j}$, $i \in[2 n]$. Since $\boldsymbol{\pi}$ is an approximate equilibrium, the priceregulating market over $\left\{G_{2 j-1}, G_{2 j}\right\}$ must buy strictly more $G_{2 j}$ than $G_{2 j-1}$ to balance the deficit. This can only happen when $\pi\left(G_{2 j-1}\right)=1+\alpha$ and $\pi\left(G_{2 j}\right)=1-\alpha$. 
However, what we really need to finish the construction is $\pi\left(H_{2 j-1}\right)=1+\beta$ and $\pi\left(H_{2 j}\right)=1-\beta$ and thus, $x_{2 j-1}=0$ and $x_{2 j}=1$ and the Nash constraint is met. The big missing piece of the puzzle is then how to enforce at an approximate equilibrium the following ratio amplification:

$$
\frac{\pi\left(G_{2 j-1}\right)}{\pi\left(G_{2 i}\right)}=\frac{1+\alpha}{1-\alpha} \Rightarrow \frac{\pi\left(H_{2 j-1}\right)}{\pi\left(H_{2 j}\right)}=\frac{1+\beta}{1-\beta}
$$

It turns out that our goal can be achieved by adding carefully a chain of copies of a non-monotone market $M$, as well as linear price-regulating markets and traders who transfer money between them (like the $T_{i, j}$ 's above). We create such a chain that starts from $G_{2 j-1}, G_{2 j}$ and ends at $H_{2 j-1}, H_{2 j}$, for each $j \in[n]$. These non-monotone markets, working with price-regulating markets, can step-by-step amplify the ratio of two goods, either from $(1+\alpha) /(1-\alpha)$ to $(1+\beta) /(1-\beta)$; or from $(1-\alpha) /(1+\alpha)$ to $(1-\beta) /(1+\beta)$. The tricky part is that all actions happen in a local neighborhood of $M$, where the phenomenon of non-monotonicity appears.

\subsection{Preparation}

Given a $2 n \times 2 n$ polymatrix game $\mathbf{P}$, we can normalize $\mathbf{P}$ to get $\mathbf{P}^{\prime}$ as follows: For all $i \in[2 n]$ and $j \in[n]$, set

$$
P_{i, 2 j-1}^{\prime}=(1+t) / 2 \text { and } P_{i, 2 j}^{\prime}=(1-t) / 2
$$

where $t=P_{i, 2 j-1}-P_{i, 2 j}$. Now all entries of $\mathbf{P}^{\prime}$ are between 0 and 1 , and $\mathbf{P}^{\prime}$ satisfies $P_{i, 2 j-1}^{\prime}+P_{i, 2 j}^{\prime}=1$, for all $i, j$. By the definition, it is easy to show that $\mathbf{P}^{\prime}$ has the same set of $\epsilon$-well-supported Nash equilibria as $\mathbf{P}$. So from now on, we assume, without loss of generality, that $\mathbf{P}$ is normalized.

Next we show that given $M$ and $\boldsymbol{\pi}$ that satisfy conditions of Theorem 6, one can normalize it to get the following more convenient form. In this extended abstract, we assume the number of goods in $M$ is $k=2$ to simplify the presentation. The proof for general $k$ can be found in the full version.

Lemma 3.1. (Normalized Non-Monotone Market) There are two (not necessarily rational) positive constants $c$ and $d$ with the following property. Given any positive and rational $\mu$ and $\gamma$, one can build a market $M_{\mu, \gamma} \in \mathcal{M}_{\mathcal{U}}$ with two goods $G_{1}$ and $G_{2}$, in time polynomial in $1 / \gamma$ and the number of bits to encode $\mu$. Let $f_{\mu, \gamma}(x)$ denote the excess demand function of $G_{1}$, when $G_{1}$ is priced at $1+x$ and $G_{2}$ is priced at $1-x$. Then $f_{\mu, \gamma}$ is well defined over $[-c, c]$; $\left|f_{\mu, \gamma}(0)\right| \leq \mu \gamma ; f_{\mu, \gamma}^{\prime}(0)=\mu d>0 ;$ and satisfies

$$
\left|f_{\mu, \gamma}(x)-f_{\mu, \gamma}(0)-\mu d x\right| \leq|x / D|, \text { for all } x \in[-c, c],
$$

where $D=\max \{20,20 / d\}$.

\subsection{Our Construction}

First of all, the two main building blocks of $M_{\mathbf{P}}$ are

Normalized Non-Monotone Markets: Given two positive rational numbers $\mu$ and $\gamma$, we use $\mathbf{n m}\left(\mu, \gamma, G_{1}, G_{2}\right)$ to denote the creation of a new copy of $M_{\mu, \gamma}$ over $G_{1}$ and $G_{2}$. Price-Regulating Market: Given positive rational numbers $\tau$ and $\alpha$, we use $\operatorname{pr}\left(\tau, \alpha, G_{1}, G_{2}\right)$ to denote the creation of a price-regulating market of parameters $\tau, \alpha$ over $G_{1}, G_{2}$.

All other traders in $M_{\mathbf{P}}$ are single-minded: each of them is interested in one specific good and spends all the budget on it. We say a trader is a $\left(\tau, G_{1}: G_{2}\right)$-trader if her endowment consists of $\tau$ units of $G_{1}$ and she is only interested in $G_{2}$; and we say a trader is a $\left(\tau, G_{1}, G_{2}: G_{3}\right)$-trader if her endowment consists of $\tau$ units of $G_{1}, G_{2}$ each and is interested in $G_{3}$.

Without loss of generality, assume $n=2^{t}$ is a power of 2 . Then $M_{\mathbf{P}}$ consists of the following $O(n t)=O(n \log n)$ many goods: $\operatorname{AUX}_{i}$ for $i \in[n]$; and $G_{i, j}$ for $i \in[2 n], j \in[0: 4 t]$.

We also divide the goods, except the $\operatorname{AUX}_{i}$ 's, into $n(4 t+1)$ groups $\left\{\mathcal{R}_{i, j}\right\}=\left\{G_{2 i-1, j}, G_{2 i, j}\right\}, i \in[n]$ and $j \in[0: 4 t]$.

Next we list all the parameters we use in the construction. We use $\alpha_{i}$ to denote $2^{i} / n^{5}$ for each $i \in[0: 4 t]$, so $\alpha_{0}=1 / n^{5}$ and $\alpha_{4 t}=1 / n$. Recall the positive constant $d$ from Lemma 3.1. We let $d^{*}$ denote a positive rational number (a constant) that satisfies $1-1 / D \leq d^{*} d \leq 1$. Other parameters we use include: $\beta=\alpha_{4 t}=1 / n, \mu=\bar{d}^{*} n=\Theta(n), \tau=n^{2}, \gamma=1 / n^{6}$, $\xi=\delta n t, \delta=\epsilon t$, and $\epsilon=1 / n^{8}$.

Construction of $M_{\mathbf{P}}$. First, we use $\mathbf{n m}$ and pr to build a closed economy over each of the $n(4 t+1)$ groups $\mathcal{R}_{i, j}$. Here by a closed economy over a group of goods, we mean a set of traders whose endowments consist of goods from this group only and they are interested in goods from this group only.

For each group $\mathcal{R}_{i, j}, i \in[n]$ and $j \in[4 t]$, we add a market $\operatorname{pr}\left(\tau, \alpha_{j}, G_{2 i-1, j}, G_{2 i, j}\right)$. We also add a non-monotone market $\mathbf{n m}\left(\mu, \gamma, G_{2 i-1, j}, G_{2 i, j}\right)$. We will refer to them simply as the pr market and the $\mathbf{n m}$ market over $\mathcal{R}_{i, j}$, respectively.

For each group $\mathcal{R}_{i, 0}$ of $\left\{G_{2 i-1,0}, G_{2 i, 0}\right\}$, where $i \in[n]$, we only add $\operatorname{pr}\left(\tau, \alpha_{0}, G_{2 i-1,0}, G_{2 i, 0}\right)$.

Next we create single-minded traders who trade between different groups. The initial endowment of each such trader consists of goods from $\mathcal{R}_{i, j}$ and is only interested in one of the goods from another group $\mathcal{R}_{i^{\prime} . j^{\prime}}$. We will refer to her as a trader who trades from $\mathcal{R}_{i, j}$ to $\mathcal{R}_{i^{\prime}, j^{\prime}}$.

At the same time we construct a weighted directed graph $\mathcal{G}$ which will be used in the proof of correctness only. Here each $\mathcal{R}_{i, j}$ corresponds to a vertex. We add an edge from $\mathcal{R}_{i, j}$ to $\mathcal{R}_{i^{\prime}, j^{\prime}}$ in $\mathcal{G}$ whenever we create a set of traders who trade from $\mathcal{R}_{i, j}$ to $\mathcal{R}_{i^{\prime}, j^{\prime}}$. Our construction below guarantees that whenever we create traders who trade from $\mathcal{R}_{i, j}$ to $\mathcal{R}_{i^{\prime}, j^{\prime}}$, the total endowment of these traders must consist of same amount, say $w>0$, of $G_{2 i-1, j}$ and $G_{2 i, j}$. We set $w$ to be the weight of this edge. We show by the end of the construction that $\mathcal{G}$ is strongly connected and for every group, its total in-weight is the same as its total out-weight.

Here is the construction: For each $i \in[2 n]$ we will use below $G_{i}$ to denote $G_{i, 0}$; and $H_{i}$ to denote $G_{i, 4 t}$. We add to $M_{\mathbf{P}}$ a $\left(P_{i, j}, H_{i}: G_{j}\right)$-trader, for each pair $i, j \in[2 n]$. As $\mathbf{P}$ is normalized, it is easy to verify that we should add an edge in $\mathcal{G}$ from $\mathcal{R}_{i, 4 t}$ to $\mathcal{R}_{j, 0}$ with weight 1 , for each $i, j$. Next, for each pair $i \in[n]$ and $j \in[0: 4 t-1]$, we add two traders: one $\left(n, G_{2 i-1, j}: G_{2 i-1, j+1}\right)$-trader and one $\left(n, G_{2 i, j}: G_{2 i, j+1}\right)$ trader. We add an edge from $\mathcal{R}_{i, j}$ to $\mathcal{R}_{i, j+1}$ of weight $n$.

This finishes the construction of graph $\mathcal{G}$. It is clear that $\mathcal{G}$ satisfies both conditions we promised earlier.

Finally, we add traders between $\operatorname{AUX}_{j}$ and $\mathcal{R}_{j, 0}$. Let

$$
r_{j}=2 n-\sum_{i \in[2 n]} P_{i, j}>0, \text { for each } j \in[2 n] .
$$

As $\mathbf{P}$ is normalized, $r_{2 j-1}+r_{2 j}=2 n$. We finally add to $M_{\mathbf{P}}$ the following three traders: one $\left((1-\beta) r_{2 j-1}, \mathrm{AUX}_{j}: G_{2 j-1}\right)$ trader, one $\left((1-\beta) r_{2 j}, \operatorname{AUX}_{j}: G_{2 j}\right)$-trader and one $((1-\beta) n$, $\left.G_{2 j-1}, G_{2 j}: \mathrm{AUX}_{j}\right)$-trader. This finishes the construction.

\subsection{Proof of Correctness}

First we introduce additively approximate market equilibria to simplify the presentation: We say $\boldsymbol{\pi}$ is an $\epsilon$-additively 
approximate market equilibrium of a market, for some $\epsilon \geq 0$, if there exists a vector $\mathbf{z} \in Z(\boldsymbol{\pi})$ such that $z_{j} \leq \epsilon$, for all $j$. We prove in the rest of this section that given an $\epsilon$-additively approximate equilibrium $\boldsymbol{\pi}$ of $M_{\mathbf{P}}$, where $\epsilon=1 / n^{8}$, we can compute a $(1 / n)$-well-supported equilibrium $\mathbf{x}$ of $\mathbf{P}$ in polynomial time. Theorem 6 follows since the total endowment of each good in $M_{\mathbf{P}}$ is $O\left(n^{2}\right)$.

In the rest of the section, we let $\boldsymbol{\pi}$ denote an $\epsilon$-additively approximate equilibrium of $M_{\mathbf{P}}$ with $\epsilon=1 / n^{8}$, and we use $\pi(G)$ to denote the price of a good $G$ in $\pi$. For each group $\mathcal{R}_{i, j}$, we let $\pi_{i, j}=\pi\left(G_{2 i-1, j}\right)+\pi\left(G_{2 i, j}\right)$. Let

$$
\begin{aligned}
& \pi_{\max }=\max \left\{\pi_{i, j}: i \in[n], j \in[0: 4 t]\right\} \text { and } \\
& \pi_{\min }=\min \left\{\pi_{i, j}: i \in[n], j \in[0: 4 t]\right\} .
\end{aligned}
$$

We also use $a=b \pm c$, where $c>0$, to denote the inequality $b-c \leq a \leq b+c$. All missing proofs below can be found in the full version. First, from the pr markets, we have

Lemma 1. For all $i \in[n]$ and $j \in[0: 4 t]$, we have

$$
\frac{1-\alpha_{j}}{1+\alpha_{j}} \leq \frac{\pi\left(G_{2 i-1, j}\right)}{\pi\left(G_{2 i, j}\right)} \leq \frac{1+\alpha_{j}}{1-\alpha_{j}}
$$

Since only one trader is interested in $\mathrm{AUX}_{j}$, we have

LEMMA 2. If we scale $\boldsymbol{\pi}$ so that $\pi_{j, 0}=2$ for some $j \in[n]$, then we have $\pi\left(\mathrm{AUX}_{j}\right) \geq 1-O(\epsilon / n)$.

Using the strong connectivity of $\mathcal{G}$ and the property that each vertex in $\mathcal{G}$ has the same total in- and out-weight, we can relate the money flowing in and out of each $\mathcal{R}_{i, j}$ to show:

LEMMA 3. If we scale $\boldsymbol{\pi}$ so that $\pi_{\min }=2$, then we have $\pi_{\max }=2+O(\epsilon t)$.

From Lemma 3, we can also upper bound $\pi\left(\mathrm{AUX}_{j}\right)$ :

LEMma 4. If we scale $\boldsymbol{\pi}$ so that $\pi_{j, 0}=2$ for some $j \in[n]$, then we have $\pi\left(\mathrm{AUX}_{j}\right) \leq 1+O(\epsilon t)$.

From now on, $\pi$ is scaled so that $\pi_{\min }=2$. Combining Lemmas 2, 3 and 4, we have $\pi\left(\operatorname{AUX}_{i}\right)=1 \pm O(\epsilon t)$ and

$$
2 \leq \pi_{i, j}=\pi\left(G_{2 i-1, j}\right)+\pi\left(G_{2 i, j}\right) \leq 2+O(\epsilon t)
$$

for all $i$ and $j$. Now we know that all prices are pretty close to each other. By Walras' law, we can show that the excess demand of each good must be close to 0 from both sides:

LEMmA 5. If $\boldsymbol{\pi}$ is an $\epsilon$-additively approximate equilibrium of $M_{\mathbf{P}}$, then there is a $\mathbf{z} \in Z(\boldsymbol{\pi})$ such that $\|\mathbf{z}\|_{\infty} \leq O(\epsilon n t)$.

Let $\xi=\epsilon n t=\log n / n^{7}$ and $\delta=\epsilon t$.

Now we are ready to recover a $(1 / n)$-well-supported Nash equilibrium of $\mathbf{P}$. Recall that we use $H_{i}$ to denote $G_{i, 4 t}$. For each $i \in[n]$, let $\theta_{i}=\left(\pi\left(H_{2 i-1}\right)+\pi\left(H_{2 i}\right)\right) / 2$ and set $\mathbf{x}$ to be

$x_{2 i-1}=\frac{\pi\left(H_{2 i-1}\right)-(1-\beta) \theta_{i}}{2 \beta \theta_{i}}$ and $x_{2 i}=\frac{\pi\left(H_{2 i}\right)-(1-\beta) \theta_{i}}{2 \beta \theta_{i}}$

where $\beta=\alpha_{4 t}=1 / n$. We have $x_{2 i-1}+x_{2 i}=1$. Also $x_{i} \geq 0$ follows from Lemma 1 . To finish the proof, we prove

TheOREM 8. When $n$ is sufficiently large, $\mathbf{x}$ built above is a $(1 / n)$-well-supported Nash equilibrium of $\mathbf{P}$.

To prove Theorem 8, we need the following key lemma.
Lemma 6. For all $i \in[n]$ and $j \in[4 t]$, we have

$$
\begin{aligned}
& \frac{1+\alpha_{j-1}}{\pi\left(G_{2 i-1, j-1}\right)}=\frac{1-\alpha_{j-1}}{\pi\left(G_{2 i, j-1}\right)} \Rightarrow \frac{1+\alpha_{j}}{\pi\left(G_{2 i-1, j}\right)}=\frac{1-\alpha_{j}}{\pi\left(G_{2 i, j}\right)} \\
& \frac{1-\alpha_{j-1}}{\pi\left(G_{2 i-1, j-1}\right)}=\frac{1+\alpha_{j-1}}{\pi\left(G_{2 i, j-1}\right)} \Rightarrow \frac{1-\alpha_{j}}{\pi\left(G_{2 i-1, j}\right)}=\frac{1+\alpha_{j}}{\pi\left(G_{2 i, j}\right)}
\end{aligned}
$$

Before proving Lemma 6, we use it to prove Theorem 8:

Proof Sketch of Theorem 8. Assume that the vector $\mathbf{x}$ we get violates one of the Nash constraints. Wlog, assume that $\mathbf{x}^{T} \cdot \mathbf{P}_{1}>\mathbf{x}^{T} \cdot \mathbf{P}_{2}+1 / n$ and we need to prove $x_{2}=0$.

Let $T_{1}, T_{2}$ denote the traders in the pr market over $\mathcal{R}_{1,0}$. To prove $x_{2}=0$, we compare the money spent by all traders in $M_{\mathbf{P}}$ on $G_{1}$ and $G_{2}$ except $T_{1}$ and $T_{2}$, which we denote by $M_{1}$ and $M_{2}$, respectively. A careful analysis combined with $\mathbf{x}^{T} \cdot \mathbf{P}_{1}>\mathbf{x}^{T} \cdot \mathbf{P}_{2}+1 / n$ implies $M_{1} \geq M_{2}+\Omega(\beta / n)$. So the difference between their demand for $G_{1}$ and $G_{2}$ is

$$
\frac{M_{1}}{\pi\left(G_{1}\right)}-\frac{M_{2}}{\pi\left(G_{2}\right)} \geq \frac{M_{2}+\Theta(\beta / n)}{\pi\left(G_{1}\right)}-\frac{M_{2}\left(1+\alpha_{0}\right)}{\pi\left(G_{1}\right)\left(1-\alpha_{0}\right)}=\omega(\xi)
$$

Because the total supply of $G_{1}$ is the same as that of $G_{2}$, it follows from Lemma 5 that the total demand of $G_{1}$ from $T_{1}, T_{2}$ must be strictly smaller than the total demand of $G_{2}$ from them. But this can only happen when $\pi\left(G_{1}\right) / \pi\left(G_{2}\right)=$ $\left(1+\alpha_{0}\right) /\left(1-\alpha_{0}\right)$. Using Lemma 6 and induction, we finally get $\pi\left(H_{1}\right) / \pi\left(H_{2}\right)=(1+\beta) /(1-\beta)$ and thus, $x_{2}=0$.

Finally, we prove Lemma 6 . Given a group $\mathcal{R}_{i, j}$, where $i$ $\in[n]$ and $j \in[4 t]$, we first scale $\pi$ again so that $\pi_{i, j}=2$. Note that what we need to prove in Lemma 6 remains the same after scaling. We are interested in the total demand of $G_{2 i-1, j}$ from all the traders in $M_{\mathbf{P}}$ except those two traders in the pr market over $\mathcal{R}_{i, j}$.

First, for the $\mathbf{n m}$ market over $\mathcal{R}_{i, j}$, we use $f(x)$ to denote the excess demand (within the $\mathbf{n m}$ market only) for $G_{2 i-1, j}$ when $\pi\left(G_{2 i-1, j}\right)=1+x$ and $\pi\left(G_{2 i, j}\right)=1-x$. Note that $f$ is $f_{\mu, \gamma}$ in Lemma 3.1 and satisfies $|f(0)|=O(\mu \gamma)$ and

$$
|f(x)-f(0)-\mu d x| \leq|\mu x / D|, \text { for all } x \in[-c, c],
$$

where $D=\max \{20,20 / d\}$ and $c>0$ are both constants independent of $n$. So when $n$ is sufficiently large, $\beta \ll c$.

Next we let $h(x, y)$ denote the excess demand function of $G_{2 i-1, j}$ from all traders except those two in the pr market over $\mathcal{R}_{i, j}$ when $\pi\left(G_{2 i-1, j-1}\right)=1+y, \pi\left(G_{2 i-1, j}\right)=1+x$ and $\pi\left(G_{2 i, j}\right)=1-x$. By Lemma 1 and 3, we are only interested in $x, y$ satisfying $|x| \leq \alpha_{j}$ and $|y| \leq \alpha_{j-1}+O(\delta)$. We have

$$
h(x, y)=f(x)-n x /(1+x)+n y /(1+x) .
$$

By (5), we get the following lemma:

Lemma 7. For any $x, y$ with $|x| \leq 3|y|$ and $|y|=\alpha_{j-1} \pm$ $O(\delta)$, we have $h(x, y)>n y / 2$ if $y>0 ;$ and $h(x, y)<n y / 2$ if $y<0$.

We are now ready to prove Lemma 6 :

Proof of Lemma 6. We first scale $\pi$ so that $\pi_{i, j}=2$. Depending on whether

$\frac{1+\alpha_{j-1}}{\pi\left(G_{2 i-1, j-1}\right)}=\frac{1-\alpha_{j-1}}{\pi\left(G_{2 i, j-1}\right)}$ or $\frac{1-\alpha_{j-1}}{\pi\left(G_{2 i-1, j-1}\right)}=\frac{1+\alpha_{j-1}}{\pi\left(G_{2 i, j-1}\right)}$ we have either $y=\alpha_{j-1} \pm O(\delta)$ or $-\alpha_{j-1} \pm O(\delta)$ by Lemma 1 and Lemma 3. Moreover, from Lemma 1 we have $|x| \leq \alpha_{j}$ 
and thus, $|x| \leq 3|y|$ since $\alpha_{j}=2 \alpha_{j-1}=\omega(\delta)$. We conclude from Lemma 7 that either $h(x, y)>n y / 2$ or $h(x, y)<n y / 2$ respectively. By $n \alpha_{j-1} \geq n \alpha_{4 t} \gg \xi$, Lemma 5 implies that the excess demand of $G_{2 i-1, j}$, within the pr over $\mathcal{R}_{i, j}$, must be either strictly negative or strictly positive, respectively.

When it is strictly negative, we know that the first trader $T_{1}$ of the price-regulating market does not spend all her budget on $G_{2 i-1, j}$. This, combined with Lemma 1, implies the first case of Lemma 6 . The other case is similar.

\section{PPAD-HARDNESS FOR CES UTILITIES}

We prove Theorem 5. Let $\rho<-1$ denote a fixed rational number and $r=-\rho$. The major challenge is that we can no longer use the linear pr markets, but only CES utilities with $\rho$. Note that we used the following two properties of $\mathbf{p r}$ markets: The price ratio is bounded between $(1-\alpha) /(1+\alpha)$ and $(1+\alpha) /(1-\alpha)$; and must be equal to one of them if the demand of $G_{1}$ from the pr market is different from that of $G_{2}$. The continuous nature of CES makes it difficult, if not impossible, to construct a market that behaves similarly.

Instead, we use the CES market $M$ of [23] (as in Example 2.2 , which is itself a non-monotone market with three isolated equilibria. The high-level picture of the construction is then similar to that of Theorem 2, in which we add to $M_{\mathbf{P}}$ a chain of copies of the non-monotone market $M$, for each $j \in[n]$, starting from $G_{2 j-1}, G_{2 j}$ and ending at $H_{2 j-1}, H_{2 j}$.

\subsection{Properties of Example 2.2}

We examine more closely the market $M$ of Example 2.2. We always assume that $\alpha$ is a positive rational number such that $a=\alpha^{1 /(r+1)}$ is rational as well. We need the following notion of excess spending: Let $S$ denote a set of traders. Given $\pi$ and a good $G$, the excess spending on $G$ from $S$ is the product of $\pi(G)$ and the excess demand of $G$ from $S$. We are interested in the excess spending $f(x)$ on $G_{1}$ from $T_{1}$ and $T_{2}$, when the prices of $G_{1}, G_{2}$ are $\pi_{1}=1+x$ and $\pi_{2}=1-x$ with $x \in(-1,1)$. Using $(2)$ we can compute a formula for $f(x)$, from which we get that $f(0)=0 ; f(x)=-f(-x)$, for any $x \in(-1,1)$; and $f^{\prime \prime}(0)=0$. Moreover, we can show:

LEMma 8. When $a>(r+1) /(r-1)$ is rational, $f^{\prime}(0)>0$ is rational and $f$ has three roots in $(-1,1)$. Let $\{-\theta, 0, \theta\}$ denote the three roots, where $\theta>0$, then we have $f^{\prime}(\theta)<0$.

From now on, we always assume that $a>(r+1) /(r-1)$, and use $\{-\theta, 0, \theta\}$ to denote the three roots of $f$ over $(-1,1)$ with $\theta>0$. Let $\lambda=f^{\prime}(0)$, a positive rational number.

Next, let $g(x)=f(x)-\lambda x$, for $x \in(-1,1)$. By definition, we have $g(0)=0, g^{\prime}(0)=0$, and $g^{\prime \prime}(0)=0$. We prove

LEMMA 9. Given any rational $r>1$, there is a rational a such that $a>(r+1) /(r-1) ; \alpha=a^{1+r}$ is rational; $g(x)$ $<0$ for all $x \in(0,1)$; and $g(x)>0$ for all $x \in(-1,0)$.

From now on, we always assume that $a$ and $\alpha$ satisfy all conditions of Lemma 9. While $\theta$ is not rational in general, we can use $f$ to get a $\gamma$-rational approximation $\theta^{*}$ of $\theta$, i.e., $\left|\theta^{*}-\theta\right| \leq \gamma$, in time polynomial in $1 / \gamma$. Let $\sigma=f^{\prime}(\theta)<0$.

Given a sufficiently large positive integer $N$, we let $A_{N}=$ $[-\delta, \delta], B_{N}=[\delta, \theta-\delta], C_{N}=[\theta-\delta, \theta+\delta], B_{N}^{\prime}=[-\theta+\delta,-\delta]$, $C_{N}^{\prime}=[-\theta-\delta,-\theta+\delta], S_{N}=[-\theta-\delta, \theta+\delta]$, where $\delta=1 / N$. We use Lemmas 8 and 9 to prove the following lemmas:

LEMma 10. When $N$ is sufficiently large, we have $|g(x)| \leq|\lambda x / 2|$ for all $x \in A_{N}$.
Lemma 11. When $N$ is sufficiently large, we have $f(x) \geq \min (\lambda,|\sigma|) \delta / 2$ for all $x \in B_{N}$.

Lemma 12. Assume that $N$ is sufficiently large. Then $g(x)=-\lambda \theta \pm \Delta$ with $\Delta=\delta(\lambda-\sigma / 2)$ implies that $x \in C_{N}$.

Note that similar lemmas also hold for $B_{N}^{\prime}$ and $C_{N}^{\prime}$.

\subsection{Our Construction}

Given a normalized $2 n \times 2 n$ polymatrix game $\mathbf{P}$, we construct a CES market $M_{\mathbf{P}}$ of parameter $\rho$ as follows.

Non-Monotone CES Markets: We use $M$ to denote the CES market discussed above, with rational constants $\alpha$ and $a$ satisfying conditions of Lemma 9. Given a positive rational number $\mu$, we use $\operatorname{ces}\left(\mu, G_{1}, G_{2}\right)$ to denote the creation of the following two traders $T_{1}$ and $T_{2}$ in $M_{\mathbf{P}}$. They are only interested in $G_{1}, G_{2}$ and have the same utilities as the two traders in $M$. $T_{1}$ has $\mu$ units of $G_{1} ; T_{2}$ has $\mu$ units of $G_{2}$.

Construction of $M_{\mathbf{P}}$. Let $m=n^{7}$. $M_{\mathbf{P}}$ has $O(n m)$ goods: $\operatorname{AUX}_{i}, G_{2 i-1, j}$ and $G_{2 i, j}$, for $i \in[n]$ and $j \in[0: m]$. We then divide them into $n(m+1)$ groups: $\mathcal{R}_{i, j}=\left\{G_{2 i-1, j}, G_{2 i, j}\right\}$.

First for each $i \in[n]$, we add a trader to $M_{\mathbf{P}}$ with $\tau=n^{4}$ units of $G_{2 i-1,0}$ and $G_{2 i, 0}$ each and her utility is $u\left(x_{1}, x_{2}\right)=$ $\left(x_{1}^{\rho}+x_{2}^{\rho}\right)^{1 / \rho}$, where $x_{1}$ (or $\left.x_{2}\right)$ denotes the amount of $G_{2 i-1,0}$ (or $G_{2 i, 0}$ ) she obtains. Next for each group $\mathcal{R}_{i, j}, i \in[n]$ and $j \in[m]$, we create $\operatorname{ces}\left(\mu, G_{2 i-1, j}, G_{2 i, j}\right)$ with $\mu=n / \lambda$.

Now we add a number of single-minded traders who trade between different groups, and follow the same notation used earlier. We define a weighted directed graph $\mathcal{G}$ similarly.

First for each $i \in[2 n]$, we use $G_{i}$ to denote $G_{i, 0}$; and $H_{i}$ to denote $G_{i, m}$. For each pair $i, j \in[2 n]$, we add to $M_{\mathbf{P}}$ one $\left(P_{i, j}, H_{i}: G_{j}\right)$-trader. Since $\mathbf{P}$ is normalized we add an edge in $\mathcal{G}$ from $\mathcal{R}_{i, m}$ to $\mathcal{R}_{j, 0}$ with weight 1 , for each $i, j \in[n]$.

Next, for each $i \in[n], j \in[m]$, we add one $\left(n, G_{2 i-1, j-1}\right.$ : $\left.G_{2 i-1, j}\right)$-trader; and one $\left(n, G_{2 i, j-1}: G_{2 i, j}\right)$-trader. We add an edge of weight $n$. This finishes the construction of $\mathcal{G}$.

Finally for each $j \in[n]$, we add traders between $\operatorname{AUX}_{j}$ and $\mathcal{R}_{j, 0}$. Let $r_{2 j-1}$ and $r_{2 j}$ be the numbers defined in (3). Let $\theta^{*}$ denote a $\gamma$-rational approximation of $\theta$, with $\gamma=1 / n^{7}$. We add one $\left(\left(1-\theta^{*}\right) r_{2 j-1}, \operatorname{AUX}_{j}: G_{2 j-1}\right)$; one $\left(\left(1-\theta^{*}\right) r_{2 j}, \operatorname{AUX}_{j}\right.$ : $\left.G_{2 j}\right)$; and one $\left(\left(1-\theta^{*}\right) n, G_{2 j-1}, G_{2 j}: \operatorname{AUX}_{j}\right)$-trader in $M_{\mathbf{P}}$.

This finishes the construction of $M_{\mathbf{P}}$.

\subsection{Proof of Correctness}

We let $\boldsymbol{\pi}$ denote an $\epsilon$-additively approximate equilibrium of $M_{\mathbf{P}}$, where $\epsilon=1 / n^{14}$. We show one can recover a $(1 / n)$ well-supported Nash equilibrium of $\mathbf{P}$ in polynomial time.

Below for each $\mathcal{R}_{i, j}$, we let $\pi_{i, j}=\pi\left(G_{2 i-1, j}\right)+\pi\left(G_{2 i, j}\right)$.

The proofs of the following three lemmas are very similar to those of Lemma 2, Lemma 3 and Lemma 4:

LEMMA 13. If we scale $\boldsymbol{\pi}$ so that $\pi_{j, 0}=2$ for some $j \in[n]$, then we must have $\pi\left(\mathrm{AUX}_{j}\right) \geq 1-O(\epsilon / n)$.

LEMma 14. Let $\pi_{\max }$ and $\pi_{\min }$ denote $\pi_{\max }=\max _{i, j} \pi_{i, j}$ and $\pi_{\min }=\min _{i, j} \pi_{i, j}$, both taken over $i \in[n]$ and $j \in[0$ : $m]$. If we scale $\boldsymbol{\pi}$ so that $\pi_{\min }=2$, then $\pi_{\max }=2+O(m \epsilon)$.

LemMA 15. If we scale $\boldsymbol{\pi}$ so that $\pi_{j, 0}=2$ for some $j \in$ $[n]$, then we have $\pi\left(\mathrm{AUX}_{j}\right) \leq 1+O(m \epsilon)$.

For each $i \in[n], j \in[0: m]$, we let $x_{i, j}$ denote the number satisfying $\left(1+x_{i, j}\right) /\left(1-x_{i, j}\right)=\pi\left(G_{2 i-1, j}\right) / \pi\left(G_{2 i, j}\right)$. Note that $x_{i, j}$ is unique, and invariant under scaling of $\boldsymbol{\pi}$. 
It would be great if we can show something similar to Lemma 5. However, right now we have no bound on the ratio of $\pi\left(G_{2 i-1, j}\right)$ and $\pi\left(G_{2 i, j}\right)$. Next we prove the following:

LemMa 16. $\left|x_{i, 0}\right|=O\left(1 / n^{3}\right)$ for all $i \in[n]$.

Let $N=n^{6}$ and thus, $x_{i, 0} \in S_{N}$. We can use the following lemma to show that $x_{i, j} \in S_{N}$ for all $i$ and $j$, by induction.

Lemma 17. For any $i \in[n]$ and $j \in[m]$, if $x_{i, j-1} \in A_{N}$, then we have $x_{i, j} \in A_{N} \cup B_{N} \cup B_{N}^{\prime}$; if $x_{i, j-1} \in B_{N}$, then $x_{i, j} \in B_{N} \cup C_{N}$; if $x_{i, j-1} \in B_{N}^{\prime}$ then $x_{i, j} \in B_{N}^{\prime} \cup C_{N}^{\prime}$; if $x_{i, j-1} \in C_{N}$, then $x_{i, j} \in C_{N}$; and if $x_{i, j-1} \in C_{N}^{\prime}$, then $x_{i, j} \in C_{N}^{\prime}$.

The proof of this lemma heavily uses properties of $f$ and $g$ proved in Section 4.1.

We construct a vector $\mathbf{y}$ from $\boldsymbol{\pi}$ as follows. Recall $\theta^{*}$ is a $\gamma$-rational approximation of $\theta$ with $\gamma=1 / n^{7}$. Let $\delta=1 / N$.

For each $i \in[n]$, if $x_{i, m} \geq \theta^{*}-2 \delta$, then we set $y_{2 i-1}=1$ and $y_{2 i}=0$; if $x_{i, m} \leq-\left(\theta^{*}-2 \delta\right)$, then we set $y_{2 i-1}=0$ and $y_{2 i}=1$; otherwise, we set $y_{2 i-1}$ and $y_{2 i}$ to be

$$
y_{2 i-1}=\left(\theta^{*}+x_{i, m}\right) /\left(2 \theta^{*}\right) \text { and } y_{2 i}=\left(\theta^{*}-x_{i, m}\right) /\left(2 \theta^{*}\right)
$$

Clearly $\mathbf{y}$ is a nonnegative and $y_{2 i-1}+y_{2 i}=1$. Note that when $x_{i, m} \in C_{N}$, we must have $x_{i, m} \geq \theta^{*}-2 \delta$ since $\gamma<\delta$, and hence $y_{2 i-1}=1$ and $y_{2 i}=0$. Similarly, if $x_{i, m} \in C_{N}^{\prime}$, then $y_{2 i-1}=0$ and $y_{2 i}=1$. To prove of Theorem 5 , we need

TheOREM 9. When $n$ is sufficiently large, $\mathbf{y}$ built above is a $(1 / n)$-well-supported Nash equilibrium of $\mathbf{P}$.

The idea is similar to the proof of Theorem 8 except that we need to replace Lemma 6 by the following:

Lemma 18. For each $i \in[n], x_{i, 0} \in B_{N} \cup C_{N}$ implies $x_{i, m} \in C_{N}$, and $y_{2 i-1}=1, y_{2 i}=0$. Similarly, we have $x_{i, 0} \in B_{N}^{\prime} \cup C_{N}^{\prime}$ implies $x_{i, m} \in C_{N}^{\prime}$, and $y_{2 i-1}=0, y_{2 i}=1$.

The key property for the proof of the lemma is that, when $x_{i, j-1}, x_{i, j} \in B_{N}$ for some $j$, then $x_{i, j}=x_{i, j-1}+\Omega(1 / N)$. Combining with Lemma 17, it follows that if $x_{i, 0} \in B_{N}$ then $x_{i, m} \in C_{N}$ because $m=n^{7}$ and $N=n^{6}$. The proof of the key property uses Lemmas 14 and 11 and a bound on the excess spending of $G_{2 i-1, j}$ and $G_{2 i, j}$.

Proof Sketch of Theorem 9. Assume a constraint is violated, wlog, $\mathbf{y}^{T} \cdot \mathbf{P}_{1}>\mathbf{y}^{T} \cdot \mathbf{P}_{2}+1 / n$, and we need to show $y_{2}=0$. For this purpose, we show that $x_{1,0} \in B_{N} \cup C_{N}$.

The theorem then follows from Lemma 18,

\section{MEMBERSHIP IN FIXP}

Next we prove Theorem 3. The input here is a market $M$ with $n$ traders and $m$ goods, with the endowments $w_{i, j}$ and coefficients $\alpha_{i, j}$ encoded in binary, and the CES parameters $\rho_{i}$ encoded in unary. Wlog, we will focus on prices $\boldsymbol{\pi}$ in the unit $m$-dimensional simplex $S$, with entries summing to 1 .

Let $w_{\max }=\max _{i, j}\left\{w_{i, j}\right\}, w_{\min }=\min _{i, j}\left\{w_{i, j}: w_{i, j}>0\right\}$, $\alpha_{\min }=\min _{i, j}\left\{\alpha_{i, j}: \alpha_{i, j}>0\right\}$, and $\alpha_{\max }=\max _{i, j}\left\{\alpha_{i j}\right\}$.

We use $h=\left(\alpha_{\min } w_{\min }\right) /\left(\alpha_{\max } w_{\max } 2 n m^{2}\right)$ and

$$
t=\max \left(\left\{\left\lceil 1-\rho_{i}\right\rceil: \rho_{i}<0\right\} \cup\left\{\left\lceil 1 /\left(1-\rho_{i}\right)\right\rceil: \rho_{i}>0\right\}\right)
$$

to define parameter $\mu=\left(h^{m} / m\right)^{t^{m}}$.
Given a vector $\boldsymbol{\pi} \in S$, we let $\hat{\boldsymbol{\pi}}$ denote the vector in which $\hat{\pi}_{j}=\max \left(\pi_{j}, \mu\right)$, for each $j \in[m]$. We will use the following continuous map $F: S \rightarrow S$ whose $j$ th dimension is:

$$
F_{j}(\boldsymbol{\pi})=\frac{\hat{\pi}_{j}+\max \left\{0, Z_{j}(\hat{\boldsymbol{\pi}})\right\}}{\sum_{k=1}^{m}\left(\hat{\pi}_{k}+\max \left\{0, Z_{k}(\hat{\boldsymbol{\pi}})\right\}\right)}
$$

where $Z_{j}(\hat{\boldsymbol{\pi}})$ denotes the excess demand of the $j$ th good.

To prove membership in FIXP, it suffices to show that the fixed points of $F$ coincide with the market equilibria of $M$, and that we can construct in polynomial time an algebraic circuit $C$ with operations $\{+,-, *, /, \max , \min , \sqrt[k]{ }\}, m$ inputs, and $m$ outputs which, on input $\boldsymbol{\pi}$, outputs $F(\boldsymbol{\pi})$. We prove that $F$ satisfies these conditions in the full version.

\section{MEMBERSHIP IN PPAD}

We now prove Theorem 4. We use the notation of Section 5. First, we show that one may assume without loss of generality that there is a trader who owns a positive amount of all $m$ goods and equally likes all of them. (This holds only for $\epsilon$-approximate equilibria, but not for $\epsilon$-tight.)

Let $\xi=\left(w_{\min } /(4 n m)\right)^{2}$ and let $\hat{\pi}_{j}=\max \left(\pi_{j}, \xi\right), j \in[m]$. We use the same continuous map $F$ given in (6), and we call $\boldsymbol{\pi} \in S$ a c-approximate fixed point of $F$ if $\|F(\boldsymbol{\pi})-\boldsymbol{\pi}\| \leq c$.

We show first that any $c$-approximate fixed point $\boldsymbol{\pi}$ of $F$, where $c=\xi \epsilon w_{\min } / 2$, is also an $\epsilon$-approximate equilibrium. Then we show that $F$ has two crucial properties: It is polynomially continuous, and it is approximately polynomially computable. We can then prove the theorem.

Proof Sketch of Theorem 4. It suffices to compute a $c$-approximate fixed point of $F$. We discretize $S$ into a regular simplicial decomposition in which each cell simplex has diameter $\leq \delta$, for a carefully chosen $\delta$ with number of bits polynomial in the size of the market and $\log (1 / \epsilon)$. We define an $m$-coloring on the vertices of the decomposition based on the approximate values of $F$ on these vertices. The coloring satisfies Sperner's lemma, so there must be a panchromatic simplex. Finding such a simplex is known to be in PPAD, e.g., using the method described in [21]. Finally, we prove that one of the vertices of a panchromatic simplex must be a $c$-approximate fixed point of $F$. The theorem follows.

\section{CONCLUSIONS}

This paper is a first step towards a systematic understanding of what features make the equilibrium analysis of markets computationally hard. To this end, we introduced the notion of non-monotone utilities, which covers a wide variety of important utility functions. We showed that for any family $\mathcal{U}$ of non-monotone utilities, it is PPAD-hard to compute an approximate equilibrium for a market with utilities that are drawn from $\mathcal{U}$ or are linear. Using our general approach, and a further, more customized analysis, we resolved the long-standing open problem on the complexity of CES markets when the parameter $\rho$ is less than -1 , showing that for any fixed value of $\rho<-1$, the problem of computing an approximate equilibrium is PPAD-hard.

This work raises clearly many questions. First, regarding CES functions, what is the complexity of computing (within desired precision) an actual (exact) equilibrium, is it FIXPcomplete? Second, can we dispense with the linear functions used in the general theorem, i.e., is it true that for any family $\mathcal{U}$ of non-monotone utilities, the approximate equilibrium problem is PPAD-hard for markets that use utilities from $\mathcal{U}$ 
only? For the important class of CES functions with (any) $\rho<-1$, we were able to show this, using a deeper analysis of the class of CES functions, and appropriate adaptations of the construction. Can a similar approach work in general for all non-monotone utilities?

Most ambitiously, can we obtain a dichotomy theorem that enables us to classify every family of utility functions (under standard, generally acceptable, mild assumptions for utilities) into those that can be solved efficiently and those that are apparently intractable (PPAD-hard and/or FIXP-hard)? The present paper takes a first step towards this goal.

\section{REFERENCES}

[1] K.J. Arrow, H.D. Block, and L. Hurwicz. On the stability of the competitive equilibrium, II. Econometrica, 27(1):82-109, 1959.

[2] K.J. Arrow, H. Chenery, B. Minhas, and R. Solow. Capital-labor substitution and economic efficiency. Review of Economics and Statistics, 63:225-250, 1961.

[3] K.J. Arrow and G. Debreu. Existence of an equilibrium for a competitive economy. Econometrica, 22:265-290, 1954.

[4] X. Chen, D. Dai, Y. Du, and S.-H. Teng. Settling the complexity of Arrow-Debreu equilibria in markets with additively separable utilities. In Proc. of FOCS, pages 273-282, 2009.

[5] X. Chen, X. Deng, and S.-H. Teng. Settling the complexity of computing two-player Nash equilibria. Journal of the ACM, 56(3):1-57, 2009.

[6] X. Chen and S.-H. Teng. Spending is not easier than trading: On the computational equivalence of Fisher and Arrow-Debreu equilibria. In Proc. of ISAAC, pages 647-656, 2009.

[7] B. Codenotti, B. McCune, S. Penumatcha, and K. Varadarajan. Market equilibrium for exchange CES economies: Existence, multiplicity, and computation. In Proc. of FSTTCS, pages 505-516, 2005.

[8] B. Codenotti, B. McCune, and K. Varadarajan. Market equilibrium via the excess demand function. In Proc. of STOC, pages 74-83, 2005.

[9] B. Codenotti, S. Pemmaraju, and K. Varadarajan. On the polynomial time computation of equilibria for certain exchange economies. In Proc. of SODA, 2005.

[10] B. Codenotti, A. Saberi, K. Varadarajan, and Y. Ye. Leontief economies encode nonzero sum two-player games. In Proc. of SODA, pages 659-667, 2006.

[11] C. Daskalakis, P.W. Goldberg, and C.H. Papadimitriou. The complexity of computing a Nash equilibrium. SIAM Journal on Computing, 39(1):195-259, 2009.

[12] O. de La Grandville. Economic Growth: A Unified Approach. Cambridge University Press, 2009.

[13] G. Debreu. Theory of Value. Wiley, 1959.

[14] X. Deng, C.H. Papadimitriou, and S. Safra. On the complexity of price equilibria. Journal of Computer and System Sciences, 67(2):311-324, 2003.

[15] N.R. Devanur and R. Kannan. Market equilibria in polynomial time for fixed number of goods or agents. In Proc. of FOCS, pages 45-53, 2008.

[16] N.R. Devanur, C.H. Papadimitriou, A. Saberi, and V.V. Vazirani. Market equilibrium via a primal-dual algorithm for a convex program. Journal of the ACM, 55(5):1-18, 2008.

[17] H.D. Dickinson. A note on dynamic economics. Review of Economic Studies, 22(3):169-179, 1954.

[18] B. C. Eaves. Finite solution of pure trade markets with Cobb-Douglas utilities. Mathematical Programming Studies, 23:226-239, 1985.

[19] E. Eisenberg and D. Gale. Consensus of subjective probabilities: The pari-mutuel method. Annals of Mathematical Statistics, 30(1):165-168, 1959.

[20] B. Ellickson. Competitive Equilibrium: Theory and Applications. Cambridge University Press, 1994.

[21] K. Etessami and M. Yannakakis. On the Complexity of Nash Equilibria and Other Fixed Points. SIAM Journal on Computing, 39(6):2531-2597, 2010.

[22] D. Gale. The linear exchange model. Journal of Mathematical Economics, 3:205-209, 1976.

[23] S. Gjerstad. Multiple equilibria in exchange economies with homothetic, nearly identical preferences. In $T R$ 288, Minnesota - Center for Economic Research, 1996.

[24] K. Jain. A polynomial time algorithm for computing an Arrow-Debreu market equilibrium for linear utilities. SIAM Journal on Computing, 37:303-318, 2007.

[25] S. Kakutani. A generalization of Brouwer's fixed point theorem. Duke Mathematical Journal, 8:457-459, 1941.

[26] A. Mas-Colell, M.D. Whinston, and J.R. Green. Microeconomic Theory. Oxford Press, 1995.

[27] R. R. Maxfield. General equilibrium and the theory of directed graphs. Journal of Mathematical Economics, 27(1):23-51, 1997.

[28] E.I. Nenakov and M.E. Primak. One algorithm for finding solutions of the Arrow-Debreu model. Kibernetica, 3:127-128, 1983.

[29] C.H. Papadimitriou. On the complexity of the parity argument and other inefficient proofs of existence. Journal of Computer and System Sciences, pages 498-532, 1994.

[30] T. Rutherford. Applied general equilibrium modeling with mpsge as a gams subsystem: An overview of the modeling framework and syntax. Computational Economics, 14:1-46, 1999.

[31] H. Scarf. The Computation of Economic Equilibria. Yale University Press, 1973.

[32] J.B. Shoven and J. Whalley. Applying General Equilibrium. Cambridge University Press, 1992.

[33] R.M. Solow. A contribution to the theory of economic growth. Quarterly Journal of Economics, 70(1):65-94, 1956.

[34] V.V. Vazirani. Spending constraint utilities, with applications to the adwords market. Mathematics of Operations Research, 35(2):458-478, 2010.

[35] V.V. Vazirani and M. Yannakakis. Market equilibrium under separable, piecewise-linear, concave utilities. Journal of the ACM, 58(3):1-25, 2011.

[36] L. Walras. Elements of Pure Economics, or the Theory of Social Wealth. 1874.

[37] Y. Ye. A path to the Arrow-Debreu competitive market equilibrium. Mathematical Programming, 111:315-348, 2008. 